\title{
Artigo Original / Original Paper \\ Diversidade florística em Áreas de Preservação Permanente de um plantio comercial de eucalipto no bioma Pampa, sul do Brasil
}

\author{
Floristic diversity of Areas of Permanent Preservation in an eucalyptus tree crop farm \\ in the Pampa biome, Brazil
}

\author{
Monique Caumo ${ }^{1,3,9}$, Luciana Pinto Paim ${ }^{1,4}$, Eduarda Demari Avrella ${ }^{1,5}$, Carla Roberta Orlandi ${ }^{2,6}$, \\ Elisete Maria Freitas ${ }^{2,7}$ \& Claudimar Sidnei Fior ${ }^{1,8}$
}

\begin{abstract}
Resumo
Possuidores de elevada biodiversidade, os campos do sul do Brasil vêm sendo ameaçados por diversos fatores, dentre eles, o avanço das fronteiras agrícolas. Áreas de Preservação Permanente (APPs) podem contribuir para a conservação da biota desses ecossistemas mesmo em paisagens cultivadas. O objetivo deste estudo foi descrever a diversidade florística de quatro APPs inseridas em um plantio comercial de Eucalyptus saligna. localizado em Pantano Grande, Rio Grande do Sul, no bioma Pampa. Durante um ano, excursões mensais foram realizadas utilizando o método do Caminhamento para coleta de material botânico. Foram registradas 295 espécies distribuídas em 60 famílias, as mais representativas foram Poaceae (51 spp.), Asteraceae (51 spp.), Cyperaceae (20 spp.), Fabaceae(17 spp.), Rubiaceae (12 spp.), Solanaceae (8 spp.), Malvaceae (8 spp.) e Myrtaceae (6 spp.), correspondendo 56,27\% do total. Sobre a origem, 274 espécies são nativas, quatro das quais endêmicas do Brasil e duas ameaçadas de extinção. Em relação ao hábito, as espécies herbáceas foram predominantes $(72,2 \%)$, seguido por arbustivas $(9,58 \%)$, subarbustivas $(7,87 \%)$, arbóreas $(6,16 \%)$, e trepadeiras (5,13\%). Tanto a proporção das famílias, quanto a relação das espécies identificadas corroboram com levantamentos realizados na região, denotando uma elevada riqueza específica. As APPs avaliadas apresentam elevada diversidade florística, mesmo encontrando-se parcialmente isoladas pelos talhões de plantio comercial de eucalipto.
\end{abstract}

Palavras-chave: biodiversidade, campos, conservação, levantamento florístico, sul do Brasil.

\begin{abstract}
Grasslands of southern Brazil are composed of high biodiversity and threatened by several factors, such as the advancement of agricultural frontiers. Areas of Permanent Preservation (APP) can contribute to the preservation of these environments. However, studies on their floristic composition are limited, especially about the herbaceous stratum. The objective of this study is to describe the floristic diversity of four Areas of Permanent Preservation inserted in an Eucalyptus saligna. tree crop farm in Pantano Grande, RS, located in the Pampa biome. For a year, monthly collections were performed by walking along all sampled areas. We identified 295 species distributed in 60 families, which Poaceae (51), Asteraceae (51), Cyperaceae (20), Fabaceae (17), Rubiaceae (12), Solanaceae (8), Malvaceae (8) and Myrtaceae (6) were more expressive, corresponding to $56.27 \%$ of total. About the origin, 274 species are native, four are endemic and two are endangered. Herbaceous species were predominant $(72,2 \%)$, followed by shrubs $(9,58 \%)$, sub-shrubs $(7,87 \%)$, trees $(6,16 \%)$ and vines $(5,13 \%)$. Both the proportion of the families and the relation of species agree with surveys carried out in the region, denoting a high specific richness. The APP evaluated present high floristic diversity, even though they are partially isolated by the eucalyptus forest.
\end{abstract}

Key words: biodiversity, grasslands, conservation, floristic survey, southern Brazil.

\footnotetext{
${ }^{1}$ Universidade Federal do Rio Grande do Sul - UFRGS, Depto. Horticultura e Silvicultura, Porto Alegre, RS, Brasil.

${ }^{2}$ Universidade do Vale do Taquari - UNIVATES, Centro de Ciências Biológicas e da Saúde, Lajeado, RS, Brasil.

${ }^{3}$ ORCID: < https://orcid.org/0000-0002-6115-8763>.

${ }^{4}$ ORCID: $<$ https://orcid.org/0000-0003-0779-6091>.

${ }^{5}$ ORCID: $<$ https://orcid.org/0000-0002-8819-983X>.

${ }^{6}$ ORCID: $<$ https://orcid.org/0000-0002-1677-257X>.

${ }^{7}$ ORCID: <https://orcid.org/0000-0002-9292-1557>.

${ }^{8}$ ORCID: < https://orcid.org/0000-0001-9893-081X>.

${ }^{9}$ Autor para correspondência: caumonique@gmail.com
} 


\section{Introdução}

A vegetação natural do Rio Grande do Sul (RS), na Região Sul do Brasil, é constituída de diversas fitofisionomias, incluindo formações florestais, arbustivas e campestres (Leite 2002). Os ecossistemas campestres, típicos do bioma Pampa, constituem campos subtropicais e são predominantes no estado, ocupando, originalmente, uma área de $131.041,38 \mathrm{~km}^{2}$ (Cordeiro \& Hasenack 2009). De origem relictual e derivados de um período mais seco (ca. 10.000 antes do presente), os campos se mantiveram por meio de distúrbios como o fogo e o pastejo, uma vez que o clima úmido contemporâneo potencialmente favoreceria a expansão florestal (Behling et al. 2001, 2007, 2009). Dentre as classificações atribuídas aos campos do sul do Brasil, Burkart (1975) segregou os campos do norte do Rio Grande do Sul, Paraná e Santa Catarina como "Campos do Brasil Central", incluídos no bioma Mata Atlântica, e "campos do Uruguai e sul do Brasil" correspondentes então ao bioma Pampa, o qual, no Brasil, é exclusivo do Rio Grande do Sul e ocupa aproximadamente $177 \mathrm{mil}$ $\mathrm{km}^{2}$ (IBGE 2004).

Os campos do Rio Grande do Sul apresentam elevada diversidade florística. São catalogadas 2.600 espécies campestres em geral, distribuídas em 89 famílias, número extremamente alto para um reduzido território, se comparada a outras formações semelhantes ao redor do mundo. Destas espécies, 1.620 ocorrem nos Campos do bioma Mata Atlântica e 2.150 para os Campos do bioma Pampa (Boldrini et al. 2015).

Diversos estudos fitossociológicos demonstram que a fisionomia dos campos é determinada pela dominância das gramíneas (Poaceae) (Soares et al. 2011; Rolim et al. 2014; Silva Filho et al. 2017) que em relação à riqueza, chegam a 319 espécies (Flora do Brasil 2020 2019a). Todavia, em números absolutos, essa riqueza é sobrepujada pelas compostas (Asteraceae), representadas por cerca de 348 espécies (Flora do Brasil 2020 2019b). De modo geral, tal diversidade parece ser resultante de múltiplos fatores, como a variabilidade do solo, a topografia, o regime de distribuição de chuvas, a temperatura e a disponibilidade de água presentes no território (Boldrini et al. 2015).

Apesar da elevada diversidade e das funções ecológicas que desempenha, somente em 2004 o Pampa foi reconhecido como bioma, e aos poucos seu território vem sendo ameaçado pelo avanço dos cultivos agrícolas, da silvicultura, da invasão de espécies exóticas, cultivadas ou não, e da urbanização (Overbeck et al. 2013; Brasil 2015), além de uma legislação que negligencia os campos (Pillar 2003). Com base em levantamentos realizados em 2009, estima-se que $54 \%$ de sua cobertura original foi convertida para outros usos, restando aproximadamente $63.719 \mathrm{~km}^{2}$ (Brasil 2011a).

Um dos mecanismos que podem contribuir para a preservação dos campos é a aplicação da legislação ambiental, por intermédio da lei de proteção da vegetação nativa (12.651/2012) (Brasil 2012), cujos principais instrumentos são as Áreas de Preservação Permanente (APP) e de Reserva Legal (Vélez-Martin et al. 2015). De maneira geral, ambas preveem a destinação de áreas na propriedade onde deve-se conservar a vegetação nativa e desempenham diversos objetivos, dentre eles, a preservação da biodiversidade. No caso das Áreas de Preservação Permanente, todavia, tratam-se de margens de cursos d'água, nascentes, bordas de tabuleiros ou chapadas, restingas, encostas, topos de morro e altitudes superiores a $1.800 \mathrm{~m}$, onde somente atividades de baixo impacto podem ser desenvolvidas em função de sua fragilidade (Brasil 2012). De fato, diversos estudos demonstram a importância da manutenção dessa vegetação na conservação da biodiversidade, uma vez que são ambientes dotados de elevada riqueza florística (Rodrigues \& Nave 2001; Soares \& Ferrer 2009; Venzke 2012) e abrigam a fauna local (Jacob 2003).

Segundo a Agência Gaúcha de Empresas Florestais, cerca de 748 mil hectares são destinados a áreas de preservação no Rio Grande do Sul, dos quais 286 mil são de APP (Ageflor 2017). É interesse das empresas a conservação e fiscalização rigorosa do cumprimento da legislação ambiental no intuito de manter os selos de certificação, que facilitam o acesso a mercados internacionais e funcionam como marketing positivo (Basso et al. 2011). Entretanto, tendo em vista que grande parte dos plantios florestais de eucalipto, principalmente das espécies Eucalyptus saligna Sm. e Eucalyptus grandis (Hill) Maiden, no Rio Grande do Sul localizam-se no Pampa (Ageflor 2017) e a necessidade de manejo para a manutenção das propriedades fisionômicas e ecológicas dos campos (Overbeck et al. 2009), não existem especificações sobre a recuperação de ambientes campestres na legislação ambiental atual, de forma que comumente são aplicadas técnicas inadequadas para a restauração do estrato 
herbáceo, como o plantio de mudas de espécies arbóreas (Vieira \& Overbeck 2015), tanto de espécies nativas quanto exóticas. Da mesma forma, carecem informações sobre a efetividade de preservação da biodiversidade dessas áreas, sobretudo relacionado ao efeito de borda e à fragmentação da paisagem causados pelos talhões dos plantios florestais comerciais (Vélez-Martin et al. 2015). Assim, o presente estudo teve por objetivo catalogar a diversidade florística de quatro Áreas de Preservação Permanente inseridas em plantio comercial de Eucalyptus saligna, localizado no município de Pantano Grande, RS, a fim de verificar a interferência desses fatores na composição de espécies frente à levantamentos realizados na mesma região.

\section{Material e Métodos}

A área do estudo encontra-se em uma área de transição entre os biomas Mata Atlântica e Pampa, entre as coordenadas $30^{\circ} 18^{\prime} 30^{\prime \prime}-30^{\circ} 20^{\prime} 30^{\prime \prime}$ Sul, e $52^{\circ} 30^{\prime} 30^{\prime \prime}-52^{\circ} 28^{\prime} 30^{\prime \prime}$ Oeste, na divisa das regiões fisiográficas da Depressão Central e da Serra do Sudeste. A região é caracterizada como uma área de tensão ecológica, em virtude da mistura entre florestas estacionais e campos (IBGE 2012).
Segundo Maluf (2000), o clima da região caracteriza-se como subtemperado úmido com temperatura média anual de $18,8^{\circ} \mathrm{C}$, com variação de $18,1 \mathrm{a} 22^{\circ} \mathrm{C}$; temperatura média do mês mais frio menor ou igual a $13{ }^{\circ} \mathrm{C}$ e do mês mais quente de $24,8^{\circ} \mathrm{C}$. A precipitação anual é de $1.400 \mathrm{~mm} \times$ $\mathrm{ano}^{-1}$ com balanço hídrico excedente na faixa de $0 \mathrm{a}$ $200 \mathrm{~mm}$ e déficit de 1 a $150 \mathrm{~mm}$ em breves períodos de seca no verão. O solo predominante é o Neossolo Regolítico, relacionado a afloramentos rochosos e o Argissolo Vermelho-Amarelo Distrófico, de textura arenosa, baixa fertilidade natural e boa drenagem. Em menor proporção, ocorre também o Planossolo Háplico Eutrófico, característico de áreas de várzea, relevo suave-ondulado e alta saturação por bases (Streck et al. 2008).

O estudo foi realizado em quatro Áreas de Preservação Permanente de fisionomia campestrearbustiva inseridas em um plantio florestal de Eucalyptus saligna no município de Pantano Grande, Rio Grande do Sul (Fig. 1). Pertencente à uma empresa privada, o horto florestal foi instalado no ano de 2006 sobre uma extensa área destinada à produção de grãos e à pecuária. Informações sobre as práticas desenvolvidas antes do plantio florestal são escassas, entretanto, é comum na

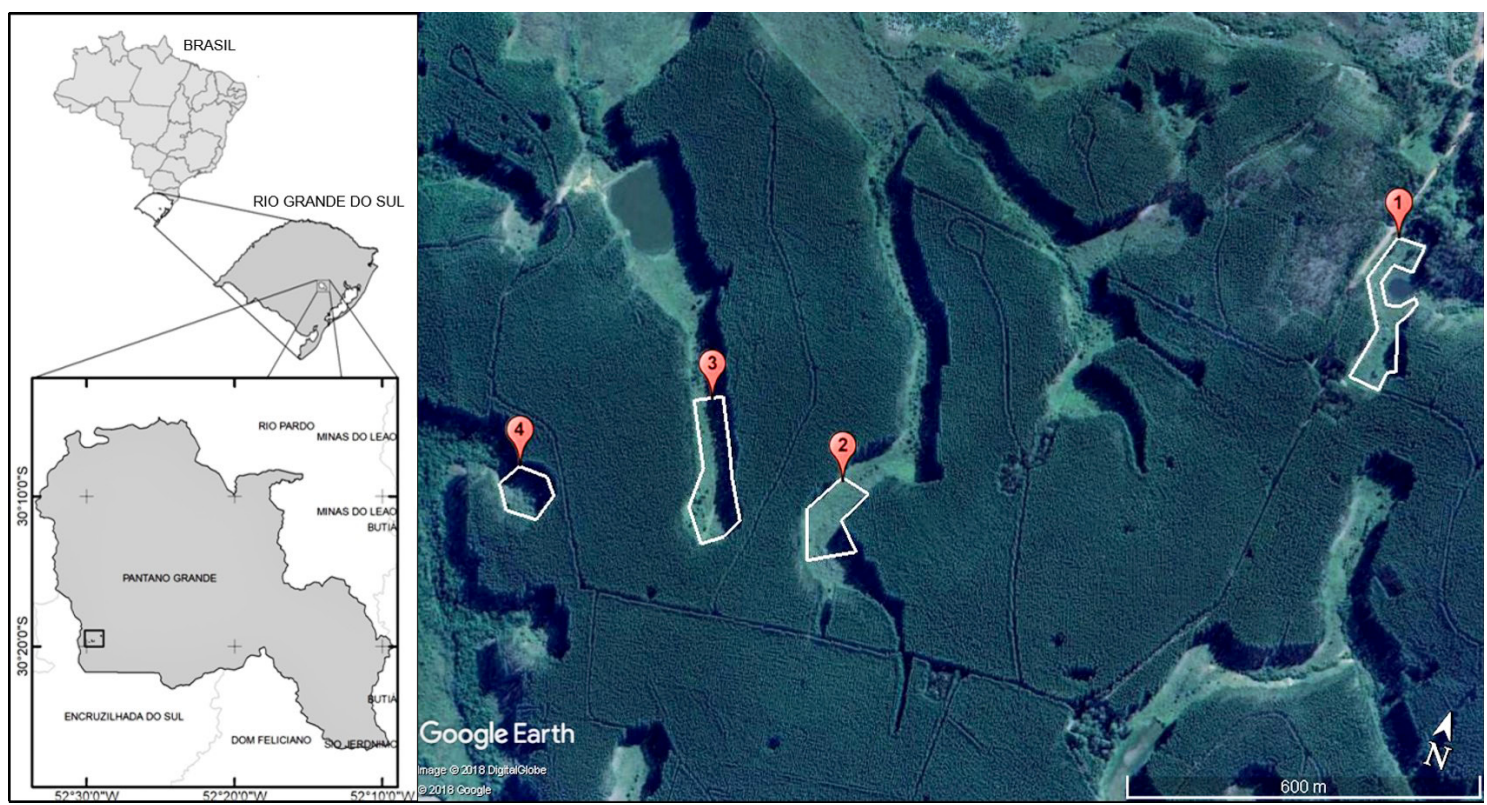

Figura 1 - Localização das áreas de estudo, inseridas no interior de um plantio comercial de Eucalyptus saligna no município de Pantano Grande, Rio Grande do Sul, bioma Pampa, Brasil. A localização das áreas de estudo em formato KML estão disponíveis para download em <https://doi.org/10.6084/m9.figshare.13093256.v1>.

Figure 1 - Location of the study areas, inserted in an Eucalyptus saligna tree crop farm in Pantano Grande, RS, Pampa biome, Brazil. The locations of the study areas in KML format are available for download at $<$ https://doi.org/10.6084/m9.figshare.13093256.v1>. 
região a realização de queimadas para controle da vegetação. A vegetação nativa presente nas APPs se reestabeleceu naturalmente, ou seja, sem a necessidade de intervenções por parte da empresa, de modo que atualmente predominam espécies arbustivas e herbáceas nas áreas mais altas. O estrato arbóreo nativo, entretanto, localizase nas porções de menor altitude de cada área e ocorre ao longo dos cursos d'água, com maior densidade conforme a proximidade ao corpo hídrico, classificado, assim, como mata de galeria (Rodrigues \& Shepherd 2000).

O horto possui 504 ha, sendo 315 destinados ao plantio, 80 ha para Reserva Legal e 97 ha para APP, dos quais, oito hectares foram analisados. Os talhões florestais distam, em média, $50 \mathrm{~m}$ do centro das áreas de preservação e, atualmente, as árvores cultivadas de Eucalyptus encontram-se com altura entre 30-35 m. A propriedade é regularmente visitada por bovinos em quantidade e tempo de permanência indetermináveis, uma vez que são inseridos por lindeiros que aproveitam a área para alimentação dos seus animais, mesmo sem o consentimento da empresa proprietária.

As Áreas de Preservação Permanente delimitam-se a partir de nascentes que ao longo de sua extensão formam pequenos córregos intermitentes, à exceção da área 1, onde existe um barramento do curso d'água, sendo todas em contato direto com o talhão de eucalipto e formações ciliares. A área 1 (30¹9'19,43’S, 52²9’02,13”O), localizada em uma região limítrofe do horto, possui 1,2 hectares em formato anguloso e é caracterizada por um relevo ondulado, onde nas seções mais altas predominam solos pedregosos (Neossolo) e nas mais baixas, solos de drenagem imperfeita (Argissolo). A área 2 (30¹9'39,75'S, $52^{\circ} 29^{\prime} 31,06^{\prime}$ 'O), de forma poligonal, compreende uma área de 1 hectare e encontra-se em uma porção interna do plantio florestal, formada por solos bem drenados e relevo suave, sendo bastante semelhante à área $3\left(30^{\circ} 19^{\prime} 39,49^{\prime} ' \mathrm{~S}, 52^{\circ} 29^{\prime} 38,78^{\prime \prime} \mathrm{O}\right)$, diferindo apenas que esta possui formato alongado e estreito. Já a área $4\left(30^{\circ} 19^{\prime} 44,31^{\prime \prime} \mathrm{S}, 52^{\circ} 29^{\prime} 49,67^{\prime \prime} \mathrm{O}\right)$ possui forma circular e área de 0,6 hectare, onde também predominam solos bem drenados e uma pequena porção de afloramento rochoso (Fig. 2). A localização das áreas em formato KML está disponível para download em $<$ https://doi. org/10.6084/m9.figshare.9936329>.

O levantamento florístico das quatro áreas de estudo foi conduzido durante o período de maio de 2016 a abril de 2017 em excursões mensais com duração de um dia, utilizando o método do Caminhamento (Filgueiras et al. 1994). Todas as áreas foram avaliadas integralmente, de forma que linhas imaginárias no sentido do maior comprimento foram traçadas e percorridas, ao mesmo tempo em que as espécies conhecidas eram registradas e as desconhecidas coletadas para posterior identificação. Foram coletados indivíduos de todos os hábitos: árvores, arbustos, subarbustos, ervas e trepadeiras. Para a identificação em laboratório, as espécies foram herborizadas e identificadas com auxílio de bibliografias específicas, comparações em herbário e consultas a especialistas. Além disso, as famílias foram classificadas segundo a The Angiosperm Phylogeny Group IV (APG IV 2016), conforme sua origem (nativas do Brasil ou exóticas) e categorizadas conforme o grau de ameaça, seguindo a Lista da Flora Gaúcha Ameaçada de Extinção (Rio Grande do Sul 2014) e o Livro Vermelho da Flora do Brasil (Martinelli \& Moraes 2013). Quando férteis, um exemplar de cada espécie foi inserido no Herbário do Vale do Taquari (HVAT) da Universidade do Vale do Taquari - Univates.

\section{Resultados e Discussão}

O inventário florístico registrou a presença de 295 espécies distribuídas em 194 gêneros pertencentes a 60 famílias (Angiospermas e Pteridófitas) (Tab. S1, disponibilizada no material suplementar <https://doi.org/10.6084/ m9.figshare.13093256.v1>). Poaceae e Asteraceae foram as famílias mais ricas em espécies nativas (51 espécies cada), seguida de Cyperaceae (20), Fabaceae (17), Rubiaceae (12), Solanaceae (8) Malvaceae (7) e Myrtaceae (6) (Fig. 3). Essas famílias juntas somam 172 espécies, o que corresponde a $56,27 \%$ do total. Das famílias amostradas, 27 foram representadas por apenas uma espécie e 10 por duas espécies. Em relação à origem, 21 espécies são exóticas, das quais 2 são consideradas invasoras (Moro et al. 2012). A tabela de espécies em formato de planilha eletrônica está disponível para download nos arquivos suplementares do artigo no link $<$ https:// doi.org/10.6084/m9.figshare.9936329>.

A representatividade das famílias amostradas (Fig. 3) coincidiu principalmente com levantamentos realizados na Depressão Central do Rio Grande do Sul, a exemplo de Boldrini \& Miotto (1987), Zoche \& Porto (1992), Boldrini (1993) e Boldrini \& Eggers (1996). Da mesma forma, as áreas avaliadas também foram similares a estudos 
florísticos desenvolvidos outras regiões do estado, como na Serra do Sudeste (Caporal \& Boldrini 2007; Girardi-Deiro et al. 1994) e na Campanha (Pinto et al. 2013). A elevada riqueza de espécies de Asteraceae é característico dos campos da região central do Rio Grande do Sul que, por sua vez, também se assemelham aos da Serra do Sudeste (Boldrini 2009).

Os gêneros com maior número de espécies são Paspalum L. e Baccharis L., com 11 e dez espécies, respectivamente, seguido de Solanum L., com sete espécies (Tab. S1, disponibilizada no material suplementar $<$ https://doi.org/10.6084/ m9.figshare.13093256.v1>). O gênero Baccharis, característico dos campos da Depressão Central (Boldrini 1997), compõe majoritariamente o estrato arbustivos das áreas, sobretudo a espécie $B$. dracunculifolia, que atua em processo de sucessão secundária em ecossistemas florestais (Oliveira \& Pillar 2004). O gênero Solanum é representado por espécies atribuídas a ambientes alterados (Mentz \&
Oliveira 2004) e podem ser associadas ao histórico de uso da área e posterior abandono, especialmente de atividades agrícolas, exceto $S$. laxum Spreng., o qual é característico de áreas de borda (Soares et al. 2008). O gênero Paspalum é de ampla distribuição geográfica, apresenta diversas espécies e alta adaptabilidade (Quarin \& Hanna 1980; Strapasson et al. 2000), de forma que na amostragem, foram observados em locais mais secos a predominância de P. plicatulum Michx., P. polyphyllum Nees e $P$. notatum Flüggé, e em manchas mais úmidas $P$. conjugatum P.J.Bergius, $P$. pumilum Nees e $P$. dilatatum Poir (Welker \& Longhi-Wagner 2007).

Considerando a localização da área de estudo como um ecótono (IBGE 2012), de maneira geral observa-se a predominância de espécies tipicamente campestres citadas em diversos levantamentos realizados no bioma Pampa, como Freitas et al. (2009), Ferreira \& Setubal (2009), Soares et al. (2011), Setubal \& Boldrini (2012), Pinto et al. (2013). Ainda, de acordo com descrição
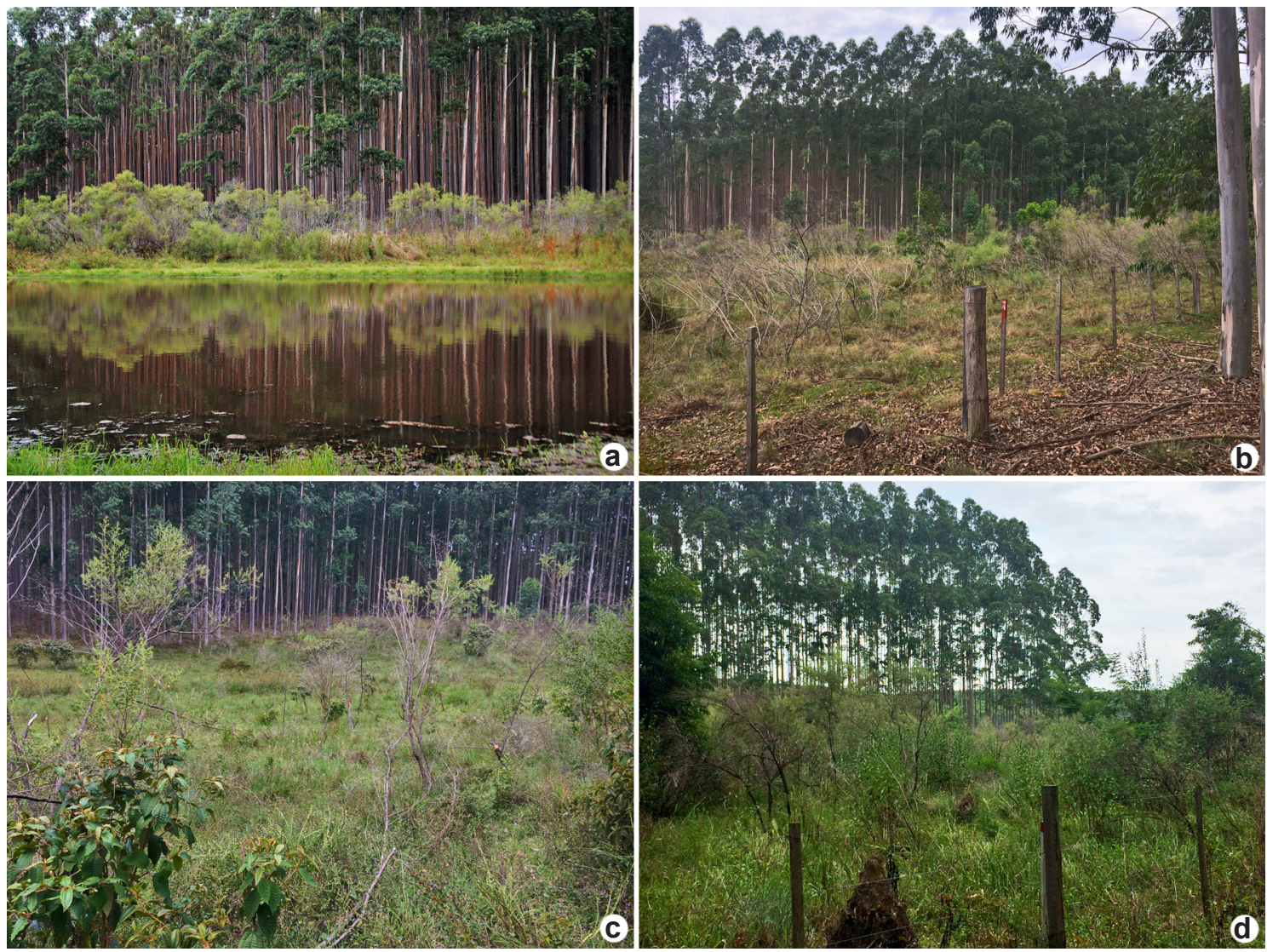

Figura 2 - a-d. Áreas de Preservação Permanente percorridas no levantamento florístico - a. área 1; b. área 2; c. área $3 ; \mathrm{d}$. área 4.

Figure 2 - a-d. Areas of Permanent Preservation covered in the floristic survey - a. area 1; b. area 2; c. area 3; d. area 4. 
de Boldrini et al. (2010), diversas espécies encontradas no estudo são características de campos do RS: P notatum e Axonopus affinis Chase de hábito prostrado e compondo o estrato inferior; Andropogon lateralis Nees, Mnesithea selloana (Hack.) de Koning \& Sosef, $P$ dilatatum de porte cespitoso destacando-se no estrato superior; leguminosas como Desmodium incanum (Sw.) DC. e Trifolium polymorphum Poir.; além de espécies da família Asteraceae, como Vernonanthura nudiflora (Less.) H.Rob., Senecio selloi (Spreng.) DC. e $S$. brasiliensis (Spreng.) Less.

A riqueza específica encontrada (295 spp.) se mostrou elevada quando considerados outros levantamentos realizados em regiões próximas, corroborando com os resultados encontrados no estudo. Boldrini (1993), no município de Eldorado do Sul/RS, bioma Pampa, encontrou 256 espécies em 30 ha avaliando quatro tratamentos de diferentes ofertas de forragem a bovinos pelo período de cinco anos. Ainda, em estudo conduzido no município de Butiá, Zoche \& Porto (1992) registraram 165 espécies em uma área de 3 hectares, confrontando o campo nativo com duas áreas de mineração, pelo período de um ano. Em região próxima, Caporal \& Boldrini (2007) em estudo desenvolvido na Serra do Sudeste sobre um campo manejado, encontraram 173 espécies em uma área de 2 hectares. Essa diferença entre a diversidade de espécies verificada na área de estudo e os demais levantamentos pode ser explicada em decorrência da maior proximidade com a Serra do Sudeste, que possui elevada heterogeneidade e diversos mosaicos vegetacionais (Rambo 1956).

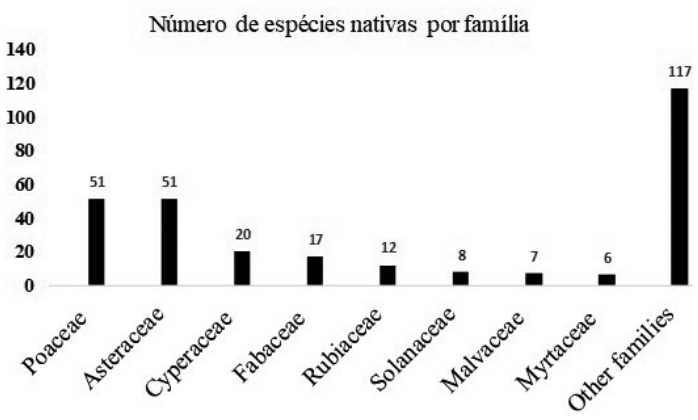

Figura 3 - Número de espécies nativas por família nas Áreas de Preservação Permanente inseridas em um plantio comercial de Eucalyptus saligna no município de Pantano Grande, RS, bioma Pampa, Brasil.

Figure 3 - Number of native species for family in Areas of Permanent Preservation in an Eucalyptus saligna tree crop farm in Pantano Grande, RS, Pampa biome, Brazil.
O estrato herbáceo é predominante e composto por diversas espécies representativas dos campos da Depressão Central, como Aristida jubata (Arechav.) Herter, A. laevis (Nees) Kunth, Eryngium horridum Malme e, principalmente, Andropogon lateralis. Em áreas mais úmidas, espécies dos gêneros Eleocharis R.Br., Fimbristylis Vahl, Rhynchospora Vahl e Cyperus L. da família Cyperaceae eram presença constante. Com relação à família Fabaceae, o levantamento registrou diversas espécies típicas desses campos, como Macroptilium prostratum (Benth.) Urb., Aeschynomene falcata (Poir.) DC., Rhynchosia diversifolia Micheli, Stylosanthes leiocarpa Vogel e, em destaque, a presença massiva de Desmodium incanum (Boldrini 1997).

Com relação à ocorrência, quatro espécies são consideradas endêmicas para o Brasil: Baccharis ochracea Spreng. (Asteraceae), Amauropelta retusa (Sw.) Pic.Serm. (Thelypteridaceae), Psidium cattleianum Sabine (Myrtaceae) e Lobelia hederacea Cham. (Campanulaceae). Em relação ao grau de ameaça de extinção das espécies registradas, Lobelia hederacea está classificada como Pouco Preocupante, segundo o Livro Vermelho da Flora do Brasil (Martinelli \& Moraes 2013). Segundo os mesmos autores, Crocanthemum brasiliensis Spach encontra-se ameaçada de extinção na categoria Em Perigo, em função de sua distribuição limitada e exposição a atividades agropastoris. Considerando a Lista de Espécies Ameaçadas do Rio Grande do Sul (Rio Grande do Sul 2014), Parodia ottonis (Lehm.) N.P.Taylor, foi registrado nas áreas de estudo e encontra-se ameaçado de extinção na categoria Vulnerável. Assim como ocorre com outras espécies da família, as causas para as ameaças são a ocorrência restrita, a conversão de hábitats, mineração e biopirataria (Brasil 2011b). Além de espécies endêmicas e ameaçadas, foram contabilizadas três espécies consideradas raras em razão da dificuldade de serem encontradas em levantamentos: Lessingianthus sellowii (Less.) H.Rob. (Asteraceae), Waltheria communis A.St.Hil. (Malvaceae) e Campomanesia aurea O.Berg (Myrtaceae) (Setubal et al. 2011).

O levantamento mostrou a predominância de espécies herbáceas (211 spp., 72,26\%), seguidas pelas espécies arbustivas (28 spp., 9,58\%); subarbustivas (23 spp., 7,87\%) arbóreas (18 spp., 6,16\%); e trepadeiras (15 spp., 5,13\%) (Fig. 4). A presença de um estrato arbustivo proeminente ocorre devido à proximidade da Serra do Sudeste, caracterizada pela ocorrência adensada de indivíduos 
do gênero Baccharis, bem como de espécies como Daphnopsis racemosa Griseb. distribuída de forma mais isolada (Boldrini 1997). As espécies arbóreas foram representadas por poucos indivíduos dispersos, tendo seus propágulos provenientes da chuva de sementes das matas de galeria adjacentes, localizadas nas áreas mais baixas e que estão em contato direto com as áreas de fisionomia campestre estudadas, realizando assim, uma conexão entre as duas formações (Müller et al. 2012). Os poucos indivíduos de hábito arbóreo inventariados no levantamento florístico encontravam-se em estágio vegetativo e em pequeno tamanho, o que poderia ser resultante da competição com as espécies herbáceas e seu sistema radicular estabelecido, da intensidade luminosa (Oliveras \& Malhi 2016), densidade de serapilheira (Marchiori 2004) e outras variáveis, dificultando, assim, o desenvolvimento e estabelecimento dessas espécies de maior porte. Além disso, a região encontra-se em uma área de tensão ecológica em que ambientes florestais e campestres, cuja estrutura e composição de espécies são distintas, ocorrem em espaços onde claramente uma formação é favorecida em detrimento da outra (Marchiori 2009).

Foram registradas 21 espécies exóticas, correspondendo a $7,1 \%$ do total de espécies registradas nas áreas do estudo. Dentre elas, Eragrostis plana Nees e Cirsium vulgare (Savi) Ten. constam como categoria 1 na lista de espécies exóticas invasoras do Rio Grande do Sul, ou seja, que têm proibido seu transporte, criação, soltura ou translocação, cultivo, propagação (por qualquer forma de reprodução), comércio, doação ou aquisição intencional sob qualquer forma (Rio

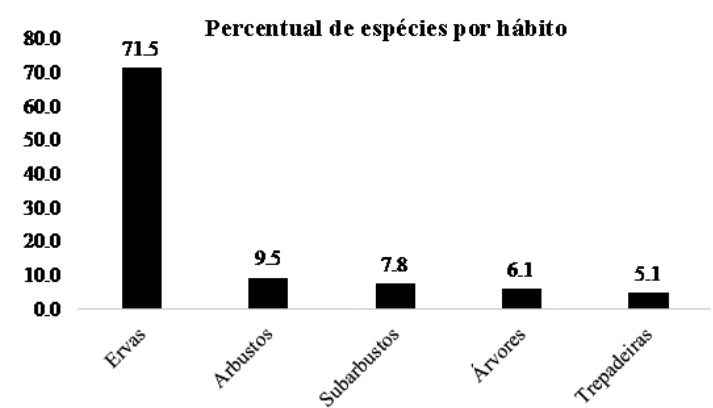

Figura 4 - Percentual de espécies por hábito nas Áreas de Preservação Permanente inseridas em um plantio comercial de Eucalyptus saligna no município de Pantano Grande, RS, bioma Pampa.

Figure 4 - Percentage of species according to life form in Areas of Permanent Preservation in an Eucalyptus saligna tree crop farm in Pantano Grande, RS, Pampa biome.
Grande do Sul 2016). De acordo com estimativas realizadas por Medeiros \& Focht (2007), existe mais de 1 milhão de hectares invadidos pelo capim annoni (E. plana) no Rio Grande do Sul, sendo detectada em diversos estudos florísticos (Quadros et al. 2003; Welker \& Longhi-Wagner 2007; Menezes et al. 2015). Cirsium vulgare, popularmente conhecido como cardo-negro, teve sua ocorrência registrada em diversos locais do RS, dentre eles no Parque Estadual do Espinilho (Galvani \& Baptista 2003), na região da Campanha (Ritter \& Baptista 2005), e no litoral (Jacobi et al. 2013). Apesar da presença das espécies nas áreas estudadas ser considerada preocupante, o percentual de cobertura registrado nas unidades amostrais ainda é baixo e exercem pouca influência sobre a diversidade local, no entanto, é o momento de realizar a sua remoção no intuito de evitar que se proliferem. Destaca-se o primeiro registro no Rio Grande do Sul de Geranium thunbergii Siebold ex Lindl. \& Paxton, uma espécie asiática comum em áreas ruderais, já encontrada em levantamentos realizados no estado de São Paulo (Aedo 2014). O histórico de uso da área envolvendo produção agrícola e pecuária, bem como os tratos envolvidos na instalação e manejo do horto florestal podem ser apontados como os principais responsáveis pela introdução dessas espécies. Da mesma forma, é comum a inserção de gado clandestinamente nas áreas de preservação, o que pode contribuir na introdução de espécies exóticas invasoras, através do transporte das sementes no trato digestivo (endozoocoria) ou quando estas ficam adpressas no corpo dos animais (epizoocoria), efetuando a dispersão (Chuong et al. 2016).

\section{Conclusão}

As Áreas de Preservação Permanente mostraram elevada diversidade de espécies, com composição florística muito semelhante aos levantamentos realizados em regiões próximas e, portanto, cumprem com sua função de conservar a flora regional. A elevada riqueza encontrada no estudo denota a importância da manutenção da vegetação nativa para a conservação, sobretudo por tratarem-se de hábitats resguardados.

Além de contribuir para o conhecimento das comunidades vegetais associadas ao estrato herbáceo de Áreas de Preservação Permanente localizadas em um bioma predominantemente campestre, a listagem das espécies fornece informações que poderão ser utilizadas em estudos de restauração ecológica, contribuindo, assim, para a conservação da flora dos campos do Rio Grande do Sul. 
Ainda, a predominância de espécies herbáceas em diferentes estádios fenológicos e características desta região bioma Pampa, reflete uma fisionomia campestre na área analisada, contrastando com a menor proporção de táxons arbóreos, os quais encontravam-se com pouco desenvolvimento.

\section{Agradecimentos}

Ao Programa de Pós-graduação em Fitotecnia da UFRGS; à Celulose Riograndense (CMPC), o apoio financeiro e logístico; à CAPES, a concessão da bolsa de Estudo; aos especialistas, o auxílio na identificação das espécies; aos colegas de departamento, o apoio na realização do levantamento.

\section{Referências}

Aedo C (2014) Novas ocorrências de Geranium L. para a flora do Brasil. Iheringia Série Botânica 69: 221-223.

Ageflor - Agência Gaúcha de Empresas Florestais (2017) A indústria de base florestal no Rio Grande do Sul. Disponível em $<$ http://www.ageflor.com.br/noticias/ wp-content/uploads/ 2017/08/A-INDUSTRIA-DEBASE-FLORESTAL-NO-RS-2017.pdf>. Acesso em 20 janeiro 2018.

APG IV - Angiosperm Phylogeny Group (2016) An update of the Angiosperm Phylogeny Group classification for the orders and families of flowering plants: APG IV. Botanical Journal of the Linnean Society 181: $1-20$.

Basso VM, Jacovine LAG, Alves RR, Valverde SR, Silva FL \& Brianezi D (2011) Avaliação da influência da certificação florestal no cumprimento da legislação ambiental em plantações florestais. Revista Árvore 35: 835-844.

Behling H, Bauermann SG \& Neves PC (2001) Holocene environmental changes from the São Francisco de Paula region, southern Brazil. Journal of South American Earth Sciences 14: 631-639.

Behling H, Pillar VD, Müller SC \& Overbeck GE (2007) Late-Holocene fire history in a forestgrassland mosaic in southern Brasil: implications for conservation. Applied Vegetation Science 10: 81-90.

Behling H, Jeske-Pieruschka V, Schüler L \& Pillar VD (2009) Dinâmica dos campos no sul do Brasil durante o Quarternário Tardio. In: Pillar VD, Müller SC, Castilhos ZMS \& Jacques AVA (eds.) Campos Sulinos: conservação e uso sustentável da biodiversidade. Ministério do Meio Ambiente, Brasília. Pp. 13-25.

Boldrini II \& Miotto STS (1987) Levantamento fitossociológico de um campo limpo da Estação Experimental Agronômica da UFRGS, Guaíba, RS. Acta Botanica Brasilica 12: 49-56.

Boldrini II (1993) Dinâmica de vegetação de uma pastagem natural sob diferentes níveis de oferta de forragem e tipos de solos, Depressão Central, Rio
Grande do Sul. Tese de Doutorado. Universidade Federal do Rio Grande do Sul, Porto Alegre. 262p.

Boldrini II \& Eggers L (1996) Vegetação campestre do sul do Brasil: dinâmica de espécies à exclusão do gado. Acta Botânica Brasilica 10: 37-50.

Boldrini II (1997) Campos do Rio Grande do Sul: caracterização fisionômica e problemática ocupacional. Boletim do Instituto de Biociências 56. Universidade Federal do Rio Grande do Sul, Porto Alegre. 39p.

Boldrini II (2009) A flora dos campos do Rio Grande do Sul. In: Pillar VD, Müller SC, Castilhos ZMS \& Jacques AVA (eds.) Campos sulinos: conservação e uso sustentável da biodiversidade. Ministério do Meio Ambiente, Brasília. Pp. 63-77.

Boldrini II, Ferreira PMA, Andrade BO, Schneider AA, Setubal RB, Trevisan R \& Freitas EM (2010) Bioma Pampa: diversidade florística e fisionômica. Ed. Pallotti, Porto Alegre. Pp. 53-62.

Boldrini II, Overbeck GE \& Trevisan R (2015) Biodiversidade de plantas. In: Pillar VD \& Lange O (eds.) Os Campos do Sul. Rede Campos Sulinos. Universidade Federal do Rio Grande do Sul, Porto Alegre. Pp. 53-62.

Brasil - Ministério do Meio Ambiente (2011a) Monitoramento do Bioma Pampa 2008-2009. Centro de Sensoriamento Remoto CSR-IBAMA, Brasília. Disponível em <https://www.mma.gov.br/ estruturas/sbf_chm_rbbio/_arquivos/relatrio_tcnico_ monitoramento_pampa_2008_2009_72.pdf $>$.Acesso em 15 novembro 2017.

Brasil - Ministério do Meio Ambiente (2011b) Plano de Ação Nacional para Conservação das Cactáceas. Série de Espécies Ameaçadas no 24. Disponível em $<$ http://www.icmbio.gov.br/portal/images/stories/ docsplanodeacao/pan_cactaceas/livro_cactaceas web.pdf $>$. Acesso em 12 outubro 2017.

Brasil - Ministério do Meio Ambiente (2015) Fifth National Report to the Convention on Biological Diversity: Brazil. Ministry of the Environment, Brasília. Disponível em <https://www.cbd.int/doc/ world/br/br-nr-05-en.pdf $>$. Acesso em 20 janeiro 2019.

Brasil (2012) Lei no 12.651, de 25 de maio de 2012. Dispõe sobre a proteção da vegetação nativa; altera as Leis nos 6.938, de 31 de agosto de 1981, 9.393, de 19 de dezembro de 1996, e 11.428, de 22 de dezembro de 2006; revoga as Leis nos 4.771, de 15 de setembro de 1965, e 7.754, de 14 de abril de 1989, e a Medida Provisória no 2.166-67, de 24 de agosto de 2001; e dá outras providências. Brasília, DF, maio de 2012. Disponível em <http://www.planalto.gov.br/ ccivil_03/ato2011-2014/2012/lei/112651.htm>. Acesso em 12 setembro 2017.

Burkart A (1975) Evolution of grasses and grasslands in South America. Taxon 24: 53-66.

Caporal FJM \& Boldrini II (2007) Florística e fitossociologia de um campo manejado na Serra do 
Sudeste, Rio Grande Do Sul. Revista Brasileira de Biociências 5: 37-44.

Cordeiro JL \& Hasenack H (2009) Cobertura vegetal atual do Rio Grande do Sul. In: Pillar VP, Müller SC, Castilhos ZMS \& Jacques AVA (eds.) Campos Sulinos: conservação e uso sustentável da biodiversidade. Ministério do Meio Ambiente, Brasília. Pp. 285-299.

Chuong J, Huxley J, Spotswood EN, Nichols L, Mariotte P \& Suding KN (2016) Cattle as dispersal vectors of invasive and introduced plants in a California Annual Grassland. Rangeland Ecology \& Management 69: 52-58.

Ferreira PMA \& Setubal RB (2009) Florística e fitossociologia de um campo natural no município de Santo Antônio da Patrulha, Rio Grande do Sul. Revista Brasileira de Biociências 72: 194-205.

Filgueiras TS, Nogueira PE, Brochado AL \& Guala IIGF (1994) Caminhamento: um método expedito para levantamentos florísticos qualitativos. Cadernos de Geociências 12: 39-43.

Flora do Brasil 2020 (2019a) Poaceae no domínio fitogeográfico Pampa Disponível em <http:// floradobrasil.jbrj.gov.br/reflora/PrincipalUC/ PrincipalUC.do?lingua=pt $>$. Acesso em 13 junho 2019.

Flora do Brasil 2020 (2019b) Asteraceae no domínio fitogeográfico Pampa. Disponível em <http:// floradobrasil.jbrj.gov.br/reflora/PrincipalUC/ PrincipalUC.do?lingua=pt $>$. Acesso em 13 junho 2019.

Freitas EM, Boldrini II, Müller SC \& Verdum R (2009) Florística e fitossociologia da vegetação de um campo sujeito à arenização no sudoeste do estado do Rio Grande do Sul, Brasil. Acta Botanica Brasilica 23: 414-426.

Galvani RF \& Baptista LRM (2003) Flora do Parque Estadual do Espinilho - Barra do Quaraí/RS. Revista da Faculdade de Zootecnia, Veterinária e Agronomia, Uruguaiana 10: 42-62.

Girardi-Deiro AM, Mota AF \& Gonçalves JON (1994) Efeito do corte de plantas lenhosas sobre o estrato herbáceo da vegetação da Serra do Sudeste, RS, Brasil. Pesquisa Agropecuária Brasileira 29: 18231832.

IBGE - Instituto Brasileiro de Geografia e Estatística (2004) Mapa de Vegetação do Brasil. IBGE, Rio de Janeiro. Mapa, colorido. Escala 1:5.000.000. Disponível em < ftp://ftp.ibge.gov.br/Cartas_e_Mapa s/Mapas_Murais/>. Acesso em 21 setembro 2017.

IBGE - Instituto Brasileiro de Geografia e Estatística (2012) Manual Técnico da Vegetação Brasileira. Série Manuais Técnicos em Geociências. IBGE, Rio de Janeiro. 275p.

Jacob AD (2003) Zonas ripárias: relações com a fauna silvestre. Anais I Seminário de Hidrologia Florestal - Zonas Ripárias. PPGEA, Florianópolis. 157p.

Jacobi SU, Duarte CI, Gonçalves RS, Acunha JS \& Hefler SM (2013) Florística dos ecossistemas do Campus
Carreiros, Rio Grande, Rio Grande do Sul, Brasil. Iheringia Série Botânica 68: 73-89.

Leite PF (2002) Contribuição ao conhecimento fitoecológico do sul do Brasil. Ciência \& Ambiente 24: 51-63.

Maluf JRT (2000) Nova classificação climática do estado do Rio Grande do Sul. Revista Brasileira de Agrometeorologia 8: 141-150.

Marchiori JNC (2004) Fitogeografia do Rio Grande do Sul - Campos Sulinos. EST, Porto Alegre. 99p.

Marchiori JNC (2009) A vegetação em Santa Maria. Ciência \& Ambiente 38: 93-112.

Martinelli G \& Moraes MA (2013) Livro Vermelho da Flora Brasileira. Andrea Jakobsson \& Instituto de Pesquisas Jardim Botânico do Rio de Janeiro, Rio de Janeiro. 1001p.

Medeiros RB \& Focht T (2007) Invasão, prevenção, controle e utilização do capim-annoni-2 (Eragrostis plana Ness) no Rio Grande do Sul, Brasil. Pesquisa Agropecuária Gaúcha 13: 105-114.

Menezes LS, Muller SC, Overbeck GE (2015) Floristic and structural patterns in South Brazilian coastal grasslands. Anais Academia Brasileira de Ciências 87: 2081-2090.

Mentz LA \& Oliveira PL (2004) O gênero Solanum na Região Sul do Brasil. Instituto Anchietano de Pesquisas, São Leopoldo. 327p.

Moro MF, Souza VC, Oliveira-Filho AT, Queiroz P, Fraga CN, Rodal MJN, Araújo FS \& Martins FR (2012) Alienígenas na sala: o que fazer com espécies exóticas em trabalhos de taxonomia, florística e fitossociologia? Acta Botânica Brasilica 26: 991-99.

Muller SC, Overbeck GE, Blanco CC, Oliveira JM \& Pillar VD (2002) South Brazilian Forest - Grassland ecotones: dynamics affected by climate, disturbance, and woody species traits. In: Myster RW (ed.) Ecotones between forest and grassland. Springer, New York. Pp. 167-87.

Oliveira JM \& Pillar VD (2004) Vegetation dynamics on mosaics of Campos and Araucaria forest between 1974 and 1999 in Southrn Brazil. Community Ecology 5: 197-202.

Oliveras I \& Malhi Y (2016) Many shades of green: the dynamic tropical forest-savannah transition zones. Philosophical Transactions of the Royal Society of London Series B 371: 20150308. < https://doi. org/10.1098/rstb.2015.0308>.

Overbeck GE, Müller SC, Fidelis A, Pfadenhauer J, Pillar VD, Blanco CC, Boldrini II, Both R \& Forneck ED (2009) Os Campos Sulinos: um bioma negligenciado. In: Pillar VD, Müller SC, Castilhos ZMS \& Jacques AVA (eds.) Campos Sulinos: conservação e uso sustentável da biodiversidade. Ministério do Meio Ambiente, Brasília. Pp. 26-41.

Overbeck GE, Hermann J, Andrade BO, Boldrini II, Kiehl K, Kirmer A, Koch C, Kollmann J, Meyer ST, Müller SC, Nabinger C, Pilger GE, Trindade JPP, VélezMartin E, Walker EA, Zimmermann DG \& Pillar VD 
(2013) Restoration ecology in Brazil - time do step out of the forest. Natureza \& Conservação - Brazilian Journal of Nature Conservation 11: 92-95.

Silva Filho PJS, Macedo RB, Vieria MS \& Neves PCP (2017) Florística e estrutura da vegetação campestre nos Campos arbustivos de São Gabriel, Rio Grande do Sul, Brasil. Iheringia Série botânica 72: 351-372.

Pillar VD (2003) Dinâmica da expansão florestal em mosaicos de floresta e campos no sul do Brasil. In: Claudino-Sales V (ed.) Ecossistemas Brasileiros: manejo e conservação. Expressão Gráfica e Editora, Fortaleza. Pp. 209-216.

Pinto MF, Nabinger C, Boldrini II, Ferreira PMA, Setubal RB, Trevisan R, Fedrigo JK \& Carassai IJ (2013) Floristic and vegetation structure of a grassland plant community on shallow basalt in southern Brazil. Acta Botanica Brasilica 27: 162-179.

Quadros FLF, Bica GS, Damé PRV, Dorow R, Kersting C \& Pötter L (2003) Levantamento das pastagens naturais da região de Santa Maria-RS, Brasil. Ciência Rural 33: 921-927.

Quarín CL \& Hanna WW (1980) Chromosome behavior, embryo sac development, and fertility of Paspalum modestum, P. boscianum, and P. conspersum. Heredity 71: 419-422.

Rambo BSJ (1956) A fisionomia do Rio Grande do Sul. Ed. Unisinos, Porto Alegre. 486p.

Rio Grande do Sul (2014) Lista da flora gaúcha ameaçada de extinção. Disponível em <http://www.fzb.rs.gov. br/conteudo/4809/?Homologada_a_nova_Lista da_Flora_Ga $\%$ C3\%BAcha_Amea $\%$ C3\% $\overline{\mathrm{A}} 7 \mathrm{ada}$ de_Extin $\%$ C3\%A7\%C3\% $\bar{A} 30>$. Acesso em $2 \overline{5}$ setembro 2017.

Rio Grande do Sul (2016) Secretaria Estadual do Meio Ambiente. Estratégias e políticas públicas para o controle das espécies exóticas invasoras. Disponível em <http://www.sema.rs.gov.br/upload/ arquivos/201706/28164322-exoticas-invasorasversaodigit al.pdf $>$. Acesso em 12 janeiro 2018.

Ritter MR \& Baptista LRM (2005) Levantamento florístico da família Asteraceae na "Casa de Pedra" e áreas adjacentes, Bagé, Rio Grande do Sul. Iheringia Série Botânica 60: 5-10.

Rodrigues RR \& Shepherd GJ (2000) Fatores condicionantes da vegetação ciliar. In: Rodrigues EE \& Leitão-Filho HF (eds.) Matas ciliares: conservação e recuperação. Editora Edusp, São Paulo. Pp. 101107.

Rodrigues RR \& Nave AG (2001) Heterogeneidade florística das matas ciliares. In: Rodrigues RR \& Leitão-Filho HF (eds.) Matas ciliares: conservação e recuperação. EDUSP/Fapesp, São Paulo. Pp. 45-71.

Rolim RG, Setubal RB, Casagrande A, Rivas MIE,
Nardin JA, Proença ML, Sandri SM, Bonilha CL \& Boldrini II (2014) Composição e estrutura de vegetação campestre em áreas com orientação norte e sul no Jardim Botânico de Porto Alegre, RS, Brasil. Iheringia, Série Botânica, Porto Alegre. Pp. 433-449.

Setubal RB, Boldrini II \& Ferreira PMA (2011) Campos dos Morros de Porto Alegre. Editora Igré Associação Sócio-Ambientalista, Porto Alegre. 243p.

Setubal RB \& Boldrini II (2012) Phytosociology and natural subtropical grassland communities in a granitic hill in southern Brazil. Rodriguésia 63: 513-524.

Soares ELC, Vignoli-Silva M, Vendruscolo GS, Thode VA, Silva JG \& Mentz LA (2008) A Família Solanaceae no Parque Estadual de Itapuã, Viamão, Rio Grande do Sul, Brasil. Revista Brasileira de Biociências 6: 177-188.

Soares AB, Carvalho PCF, Nabinger C, Trindade JPP, Trindade JK \& Mezzalira JC (2011) Dinâmica da composição botânica numa pastagem natural sob efeito de diferentes ofertas de forragem. Ciência Rural 41: 1459-1465.

Soares LR \& Ferrer RS (2009) Estrutura do componente arbóreo em uma área de floresta ribeirinha na bacia do rio Piratini, Rio Grande do Sul. Brasil Biotemas 22: 47-55.

Strapasson E, Vencovsky R \& Batista LAR (2000) Seleção de descritores na comparação de germoplasma de Paspalum spp. por meio de componentes principais. Revista Brasileira de Zootecnia 29: 373-381.

Streck EV, Kämpf N, Dalmolin RSD, Klamt E, Nascimento PC, Schneider P, Giasson E \& Pinto LFS (2008) Solos do Rio Grande do Sul. Emater/RS, Porto Alegre. $222 p$.

Vélez-Martin E, Chomenko L, Madeira MM \& Pillar VD (2015) Políticas públicas para os Campos. In: Pillar VD \& Lange O (eds.) Os Campos do Sul. Rede Campos Sulinos, Universidade Federal do Rio Grande do Sul, Porto Alegre. Pp. 169-176.

Venzke TS (2012) Florística de comunidades arbóreas no município de Pelotas, Rio Grande do Sul. Rodriguésia 63: 571-578.

Vieira MS \& Overbeck GE (2015) Recuperação dos Campos. In: Pillar VD \& Lange O (eds.) Os Campos do Sul. Rede Campos Sulinos Universidade Federal do Rio Grande do Sul, Porto Alegre. Pp. 151-156.

Welker CAD \& Longhi-Wagner HM (2007) A família Poaceae no Morro Santana, Rio Grande do Sul, Brasil. Revista Brasileira de Biociências 5: 53-92.

Zoche JJ \& Porto ML (1992) Florística e fitossociologia de campo natural sobre banco de carvão e de áreas mineradas, Rio Grande do Sul, Brasil. Acta Botanica Brasilica 62: 47-84.

Editor de área: Dr. Marcelo Moro Artigo recebido em 05/08/2018. Aceito para publicação em 18/10/2019. This is an open-access article distributed under the terms of the Creative Commons Attribution License. 